\title{
Analysis of Embryoid Bodies Derived from Human Induced Pluripotent Stem Cells as a Means to Assess Pluripotency
}

\author{
Steven D. Sheridan, ${ }^{1}$ Vasudha Surampudi, ${ }^{2}$ and Raj R. Rao ${ }^{2}$ \\ ${ }^{1}$ Center for Human Genetic Research, Massachusetts General Hospital, Harvard Medical School, Boston, \\ MA 02114, USA \\ ${ }^{2}$ Department of Chemical and Life Science Engineering, School of Engineering, Virginia Commonwealth University, \\ Richmond, VA 23284, USA \\ Correspondence should be addressed to Raj R. Rao, rrrao@vcu.edu
}

Received 3 October 2011; Accepted 29 November 2011

Academic Editor: Rajarshi Pal

Copyright $\odot 2012$ Steven D. Sheridan et al. This is an open access article distributed under the Creative Commons Attribution License, which permits unrestricted use, distribution, and reproduction in any medium, provided the original work is properly cited.

\begin{abstract}
Human induced pluripotent stem cells (hiPSCs) have core properties of unlimited self-renewal and differentiation potential and have emerged as exciting cell sources for applications in regenerative medicine, drug discovery, understanding of development, and disease etiology. Key among numerous criteria to assess pluripotency includes the in vivo teratoma assay that has been widely proposed as a standard functional assay to demonstrate the pluripotency of hiPSCs. Yet, the lack of reliability across methodologies, lack of definitive clinical significance, and associated expenses bring into question use of the teratoma assay as the "gold standard" for determining pluripotency. We propose use of the in vitro embryoid body (EB) assay as an important alternative to the teratoma assay. This paper summarizes the methodologies for creating EBs from hiPSCs and the subsequent analyses to assess pluripotency and proposes its use as a cost-effective, controlled, and reproducible approach that can easily be adopted to determine pluripotency of generated hiPSCs.
\end{abstract}

\section{Introduction}

Human induced pluripotent stem cells (hiPSCs) are developing as exciting cell sources for applications in regenerative medicine [1] and drug discovery, primarily based on their extensive similarities to their human embryonic stem cell counterparts and shared properties of self-renewal and multilineage differentiation capabilities. The strategy of inducing pluripotency by activating the pluripotent network has proven to be successful in the reprogramming of somatic cells back to an embryonic-like state [2,3]. However, there is a critical need to assess the pluripotent capabilities of the hiPSCs on a line-by-line basis once reprogramming has occurred to demonstrate this differentiation potential.

Multiple criteria have been proposed to evaluate the pluripotent state of generated hiPSCs [4-6]. Key among these criteria includes routine morphological analysis of cells for the presence of high nuclear-cytoplasmic ratio, cell surface and gene expression of pluripotent markers, demonstration of differentiation capabilities into derivatives from the three developmental germ layers (ectoderm, endoderm, and mesoderm), and specialized functional outcomes to demonstrate developmental potency. The functional assays developed thus far include in vitro differentiation, teratoma formation, chimera development, germline transmission, and tetraploid complementation [7]. The most stringent test to screen for pluripotency is the ability to demonstrate germline competency after chimera development, a test that can easily be conducted for mouse iPSCs. For hiPSCs, where testing for germline transmission and tetraploid complementation is not possible, teratoma formation of hiPSCs injected into immunocompromised mice and subsequent analysis of tissue formation has been widely used as an important methodology to investigate the developmental ability of the generated hiPSCs. 


\section{The Teratoma Assay for Pluripotency Assessment}

Teratomas are solid, defined tumors, often germ-line derived, composed of the highly organized differentiated cells and tissues containing representatives of the three developmental germ layers that can also be generated artificially by transplanting pluripotent stem cells (hESCs or hiPSCs) into immunodeficient mice [8]. The histopathology of teratomas is remarkable in that they can serve as an important tool to observe early morphogenesis into organized tissues. The number of cells transplanted into the immunodeficient animal model in order to generate the fully differentiated teratoma in vivo can range anywhere between a hundred to a million cells or more [9]. As part of this methodology, the generated hiPSCs are usually transplanted at the following sites: intramuscular, subcutaneous, under the testis capsule, or under the kidney capsule in an immune deficient mouse. After a period of at least three weeks, the mature teratomas are excised out of the animal to be assessed for the presence of the cells derived from the three germ layers. The teratoma assay has been proposed to be the most stringent means to assess pluripotency of hiPSCs [10] but is not as rigid as the tetraploid complementation and germline transmission that can be conducted with mouse iPSCs [11]. In addition, the high costs associated with the assay, use of dozens or hundreds of animals, lack of a definitive clinical or biological relevance of the ability to form teratomas to the specific cell types subsequently derived from the hiPSCs, and the nonsystematic way this assay has been employed bring into question the use of the teratoma assay as the "gold standard" $[4,6,8,11]$.

Recent reports have highlighted the need to standardize the protocol to generate and examine the teratomas induced in the immunodeficient mouse models, if they are to be used as the gold standard for assessing pluripotency in generated hiPSCs $[8,10]$. Studies have shown that some of the hiPSC lines have not been successful in forming all three germ layers in the teratomas but have been successful in deriving certain cell types that may have clinical significance [12]. Other researchers have also found that partially reprogrammed hiPSCs can form teratomas even if they do not meet other criteria for pluripotency, leading many to question the overall significance of the teratoma assay [6]. Generation of systematic protocols for teratoma generation and analysis that can be adopted across different labs also remains a challenge [8].

\section{Embryoid Bodies as a Means to Assess Pluripotency of hiPSCs}

An important alternative method to the teratoma assay is an in vitro approach involving the generation of embryoid bodies (EBs) from hiPSCs. EBs are three-dimensional aggregates of cells that are an amalgam of the three developmental germ layers [7]. In this approach, the undifferentiated hiPSCs are placed in suspension, which promotes stochastic differentiation into cells of all three germ layers. Formation of EBs is a routine approach used in the differentiation of the
hiPSCs into different cell lineages [13]. One of the major advantages of this approach is that it is performed in vitro with standard tissue culture methods and materials, thus avoiding the regulatory issues and extensive expenses associated with maintaining immune-deficient mice. The EBs can be easily grown in a suspension culture in a petri dish in the laboratory and can be scaled up without much difficulty once the appropriate conditions for scale-up are established [14]. Unlike the teratoma assay in which hiPSCs that have passed all other pluripotency tests yet fail to form teratomas for unknown reasons [12], hiPSCs readily form EBs by a number of methods providing the ability to demonstrate trilineage differentiation and analysis in a more controlled, reproducible manner. The numerous approaches that have been developed to generate EBs along with the established analyses used for their pluripotent assessment are summarized in the following sections.

\section{Methodologies for the Formation of hiPSC Embryoid Bodies}

There have been several methods developed to create embryoid bodies for a variety of purposes, from the generation of specific tissue types to stochastic in vitro germ layer differentiation, to illustrate potency of candidate pluripotent stem cell lines [13, 15-17] (summarized in Table 1). For specific tissue lineages, EBs have been shown to be beneficial in the initiation of differentiation and to enhance the differentiation towards certain lineages [18] such as hematopoietic [15, 19], neural [20,21], and cardiac tissues [22-25]. Methods for developing EBs differ in their ability to form aggregates of uniform size and the maintenance of their long-term viability. Typically, it is advantageous to control the uniform size of EBs for the reproducible differentiation of specific tissue types; however, the ability to form EBs of varied size for extended culture periods facilitates the formation of diverse tissues representing the three germ layers as a means to demonstrate differentiation potential. Both types of techniques can be used for the assessment of pluripotent stem line (hESC, hiPSC) quality.

\subsection{Heterogeneous Methods of Embryoid Body Formation.} Methods for the creation of stochastic EBs are the most straightforward and useful for the generation of varied germ layer representatives for subsequent demonstration of pluripotency [16]. Liquid suspension culture (LSC, [15]) is a common method for creating EBs and depends on the ability to grow cellular aggregates without attachment to the tissue culture vessel (Figure 1(a)). Typical tissue culture vessels for this purpose range from nontissue culture-treated petridishes to specially treated ultra-low attachment surfaces available through several vendors. These low-attachment tissue culture vessels are typically coated to provide a neutral, hydrophilic surface to prevent protein adsorption and subsequent cell attachment [26]. Hydrogels, such as naturally occurring agarose, or chemically defined, synthetic materials such as polyhydroxyethylmethacrylate (pHEMA) have been used to coat vessel surfaces to prevent the attachment of cells 
TABLE 1: Common techniques to form human embryoid bodies.

\begin{tabular}{|c|c|c|c|c|c|}
\hline & $\begin{array}{l}\text { Controls } \\
\text { EB size }\end{array}$ & $\begin{array}{l}\text { Need for single cell } \\
\text { suspension }\end{array}$ & $\begin{array}{l}\text { Large-scale bioreactor } \\
\text { production }\end{array}$ & $\begin{array}{l}\text { Need for special } \\
\text { equipment }\end{array}$ & References \\
\hline \multicolumn{6}{|l|}{ Heterogeneous methods } \\
\hline Liquid suspension culture & No & No & No & No & {$[15,16]$} \\
\hline Stirred flask culture & No & No & Yes & Yes & [27] \\
\hline Rotary cell culture systems (RCCSS) & No & No & Yes & Yes & {$[28,29]$} \\
\hline \multicolumn{6}{|l|}{ Homogeneous methods } \\
\hline Hanging drop culture & Yes & Yes & No & No & {$[13,30,31$} \\
\hline Low adhesion U-bottom multiwell plates & Yes & No & No & No & [14] \\
\hline Indented solid microsurfaces (Aggrewell ${ }^{\mathrm{TM}}$ ) & Yes & Yes & No & Yes & {$[32]$} \\
\hline \multicolumn{6}{|l|}{ Other methods } \\
\hline Hydrogels (e.g., methylcellulose, agarose, alginate) & No & Yes & No & No & {$[15,33,34$} \\
\hline
\end{tabular}

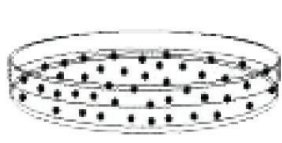

(a)

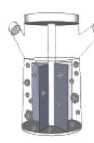

(b)

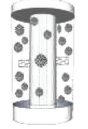

(c)

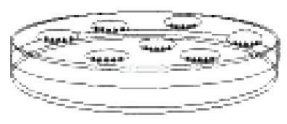

(d)

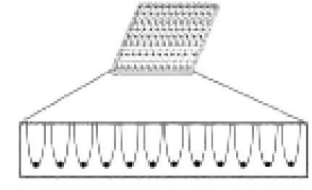

(e)

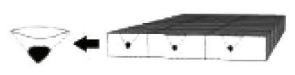

(f)

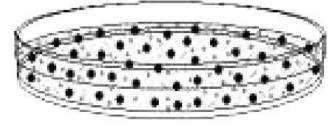

(g)

FIGURE 1: Schematic representation of methods to form embryoid bodies from pluripotent stem cells includes (a) liquid suspension culture, (b) stirred flask culture, (c) rotary cell culture systems, (d) hanging drop culture, (e) low adhesion U-bottom multiwall plates, (f) indented solid microsurfaces, and (g) hydrogel culture systems.

for the purpose of creating suspension cellular aggregates [26]. Though these surface treatments prevent the cell attachment to the tissue culture vessel resulting in aggregation, initial seeding densities need to be optimized in order to facilitate the cell-cell interactions required to form appropriately sized EBs. Seeding densities that are too low result in poor aggregation thus resulting in poorly established EB cultures. Thus, typical seeding densities are kept quite high during the initial aggregation (minimum of $10^{6}$ cells per $2 \mathrm{~mL}$ media in a $50 \mathrm{~mm}$ plate [16] or 6-well plate well). In the case of pluripotent hESC and hiPSC cultures, it is often desirable to facilitate the initial aggregation step by transferring isolated sections of colonies to low attachment vessels, since these cultures tend to be negatively effected by being in single cell suspension resulting in poor viability by apoptosis [35]. Once formed, suspension EBs cultures are typically cultured in the low attachment vessel for an extended period of time, typically from 28 to 35 days with fresh medium exchange every 2-3 days, though longer culture times may be desirable. Medium conditions have been demonstrated to have an important influence on the viability and germ layer differentiation of the suspended EBs. For instance, studies have shown that culture of EBs in a physiological glucose concentration ( $5.5 \mathrm{mM}$ as opposed to $25 \mathrm{mM}$ high glucose formulations typical of hESC and hiPSC expansion medium) in the presence of basic fibroblast growth factor (bFGF) prolonged the viability and increased the complexity of tissues in the EBs showing that representatives of all three germ layers in cultures could be maintained up to 105 days [36].

Though static suspension cultures of EBs are the most widely used due to their simplicity and the minimal cost of low-attachment treated standard vessels (dishes and multiwell plates), recent advances in bioreactor suspension culture have demonstrated usefulness of these techniques to optimize, standardize, and scale up the formation of EBs [17, 28, 29]. Unlike static suspension cultures, these systems require specialized culture equipment such as stirred flasks or the more recent Rotating Cell Culture Systems (RCCSs), developed by NASA, either the Slow Turning Lateral Vessel (STLV) or the larger capacity High Aspect Rotating Vessel (HARV) [37-39]. Typical stirred flask methodologies involve seeding hESC or hiPSC suspensions in a specialized flask utilizing a magnetic stirring bar to continually rotate the culture in order to facilitate aggregation of the cells into sustained cellular aggregates and provide better gas exchange than static systems [27] (Figure 1(b)). This method has been used to derive EBs and is amendable to scale up for larger yields [27]. Unlike these stirred flask suspension systems which allow for 
the EBs to be agitated over culture time allowing for greater gas exchange, less hypoxia, and less agglomeration than the static suspension system [27], the RCCS systems greatly reduce shear forces, present in the stirred flask systems, which can greatly damage the EBs $[40,41]$. Often termed "microgravity" cell culture, RCCS systems allow for the continuous horizontal rotation resulting in very low shear stress on the cells, active membrane-based gas diffusion to prevent hypoxia and to equally distribute both oxygen and expiration waste gas, and can partially control EB size by regulating rotation speed and initial seeding densities (Figure 1(c)). Using these RCCS systems, the STLV in particular with its high membrane surface area to medium volume compared to the larger HARV, it has been demonstrated that EBs cultured in this fashion demonstrated higher viability, more complex tissue differentiation, and, in a specific example of neural induction, enhancement of neural progenitor differentiation [42].

4.2. Homogenous Methods of Embryoid Body Formation. As opposed to the heterogeneous methods of EB formation that result in representatives of the three germ layers in a stochastic culture, it is frequently the case that more controlled EB formation methods for increased reproducibility and size control can facilitate the differentiation towards specific tissue types. In particular, control of EB size has been demonstrated to influence viability, proliferation, and differentiation potential [26] to cardiomyocytes [22, 25, 30, 43], endothelial tissue [43], as well as instruct hematopoiesis [19].

Several methods have been developed in order to form EBs of defined size. These methods share in their methodology ways to segregate a defined number of cells in order to allow them to aggregate before being collected for further culture. The hanging drop method utilizes $20-25 \mu \mathrm{L}$ drops containing a defined number of cells (typically 1000-10000) in single cell suspension $[13,30,31]$. The drops are placed onto the underside of a flipped $100 \mathrm{~mm}$ tissue culture plate lid, typically a maximum of 96 drops if using a multichannel pipette to place the drops (Figure $1(\mathrm{~d})$ ). Once placed, the lid is carefully flipped allowing the drops to remain attached to the lid and the lid is placed over a buffer-filled plate to prevent evaporation of the hanging drops. This technique is limited in the upper size limit of the initial cell aggregate due to the limited volume of the hanging drop required to allow fluid tension to adhere the drop to the lid ( 20 to $25 \mu \mathrm{L}$ ). Initial formation of the cellular aggregates typically takes 1 to 2 days, after which the EBs are individually collected manually and placed in low-adhesion plates for further culture and maturation. A variation on the hanging drop using round bottom ultra-low attachment-treated multiwall plates has also been developed which allows for the derivation of larger EBs than the hanging drop method, in that larger amounts of cells can be placed into each well (i.e., 100-200 $\mu \mathrm{L}$ of cell suspension per well in 96-well round-bottom plates) (Figure 1(e)) [14]. More recently, using a silicon wafer-based microfabrication technology containing hundreds or thousands of micrometer sized wells per $\mathrm{cm}^{2}$ adhered to the well bottoms of a standard multiwall plate, studies have demonstrated the ability to form large numbers of uniform and synchronized human EBs of defined size [32] in a commercially available format (AggreWell $^{\mathrm{TM}}$, Stem Cell Technologies) (Figure 1(f)). Though these techniques allow for the controlled aggregation of hESCs and hiPSCs, both require the formation of single cell suspensions exposing the cells to low viability and poor EB formation and, in the case of the plate based systems, centrifugation in order to force settle the cells into the bottom of the wells. It is common, therefore, that protective agents, such as the addition of Rho-associated kinase (ROCK) inhibitor, Y-27632, are required for these methods [35].

4.3. Other Embryoid Body Formation Methodologies. The EB formation methods discussed previously represent the most commonly used for the purpose of assessing hESC and hiPSC pluripotency in vitro. Other more specific approaches exist for niche applications such as encapsulation techniques utilizing hydrogels such as methylcellulose $[15,33]$ or hyaluronic acid (HA) [34]. These techniques allow for the entrapment of single cells in suspension and subsequent growth of cellular aggregates (Figure $1(\mathrm{~g})$ ). These techniques have been mostly utilized for more specific goals than pluripotency assessment such as when cell clusters derived from single-cell clones are desirable. These techniques often suffer from very low yields [15] due to the intrinsic instability of prolonged single cell culture of the pluripotent stem cells as well as complicating the isolation of EBs from the hydrogel for subsequent downstream analyses.

\section{Analysis of Germ Layer Formation in hiPSC-Derived Embryoid Bodies}

In order to assess the pluripotency of hiPSCs by the in vitro method of deriving EBs, it is imperative to have definitive downstream assays that demonstrate the ability to form representatives of the three developmental germ layers, thus demonstrating their increased differentiation potency upon reprogramming. There are several methods to show this that range from less stringent (showing expression of germ layerspecific genes) to the more stringent and definitive demonstration of tissue-similar structures (histology and immunohistochemistry) that resemble early embryonic development along with the concomitant expression of markers. This section will briefly review the typical requirements for such analyses.

5.1. Expression of Germ Layer-Specific Genes. Perhaps the least stringent test of pluripotency in EBs is the demonstration of germ layer-specific gene expression by biochemical means. The reason for the lower stringency is that this only represents the ability to detect such gene expression without knowledge of higher-order structural or temporal expression within organized structures which can be discerned by more extensive histological and immunohistochemical examination (see the following). However, it is clear that the input cells, often fibroblasts in the case of their derivation toward hiPSCs, do not express these genes and can be used as an indicator of acquired tissue specific expression [61] (i.e., neural-specific genes being expressed). Gene expression analysis 
TABLE 2: Typical markers for the analysis of human embryoid bodies.

\begin{tabular}{|c|c|c|c|c|c|c|}
\hline & Marker name & Alternate name & Marker type & $\begin{array}{l}\text { Suitable marker for } \\
\text { gene expression }\end{array}$ & $\begin{array}{l}\text { Suitable marker } \\
\text { for IHC }\end{array}$ & Reference \\
\hline \multicolumn{7}{|l|}{ Pluripotency } \\
\hline & Oct4 (POU5F1) & $\begin{array}{l}\text { Octamer-binding } \\
\text { transcription factor } 4\end{array}$ & Transcription factor & Yes & Yes & {$[44]$} \\
\hline & Nanog & $\mathrm{n} / \mathrm{a}$ & Transcription factor & Yes & Yes & {$[45]$} \\
\hline & REX-1 & $\begin{array}{l}\text { Zinc finger protein } 42 \\
\text { homolog }(Z F P 42)\end{array}$ & Transcription factor & Yes & Yes & {$[2]$} \\
\hline & SOX-2 & $\begin{array}{l}\text { SRY (sex determining } \\
\text { region Y)-box } 2\end{array}$ & Transcription factor & Yes & Yes & {$[46]$} \\
\hline & SSEA-3 & $\begin{array}{l}\text { Stage-specific embryonic } \\
\text { antigen } 3 \text { (SSEA-3) }\end{array}$ & Surface antigen & No & Yes & [47] \\
\hline & SSEA-4 & $\begin{array}{l}\text { Stage-specific embryonic } \\
\text { antigen } 3 \text { (SSEA-4) }\end{array}$ & Surface antigen & No & Yes & {$[47]$} \\
\hline & Tra-I-60 & $\mathrm{n} / \mathrm{a}$ & Surface antigen & No & Yes & [48] \\
\hline & Tra-I-81 & $\mathrm{n} / \mathrm{a}$ & Surface antigen & No & Yes & [48] \\
\hline \multicolumn{7}{|l|}{ Ectoderm } \\
\hline & GFAP & $\begin{array}{l}\text { Glial fibrillary acidic } \\
\text { protein }\end{array}$ & $\begin{array}{l}\text { Glial intermediate } \\
\text { filament }\end{array}$ & Yes & Yes & [49] \\
\hline & Nestin & $\mathrm{n} / \mathrm{a}$ & Intermediate filament & Yes & Yes & [49] \\
\hline & Pax-6 & Paired box gene 6 & Transcription factor & Yes & Yes & {$[50]$} \\
\hline & Sox-1 & $\begin{array}{l}\text { Sex determining region } \\
\text { Y-box } 1\end{array}$ & Transcription factor & Yes & Yes & [51] \\
\hline \multicolumn{7}{|l|}{ Endoderm } \\
\hline & AFP & Alpha-fetoprotein & Plasma Protein & Yes & No & [52] \\
\hline & Amylase & Alpha-amylase & Metabolic Enzyme & Yes & Yes & {$[53]$} \\
\hline & FOXA2 & $\begin{array}{l}\text { Hepatocyte nuclear } \\
\text { factor 3-beta (HNF-3B) }\end{array}$ & Transcription Factor & Yes & Yes & {$[54]$} \\
\hline & PDX1 & $\begin{array}{l}\text { Pancreatic and duodenal } \\
\text { homeobox } 1 \text {, insulin } \\
\text { promoter factor } 1\end{array}$ & Transcription factor & Yes & Yes & [55] \\
\hline & GATA4 & GATA binding protein 4 & Transcription factor & Yes & Yes & {$[56]$} \\
\hline \multicolumn{7}{|l|}{ Mesoderm } \\
\hline & Brachyury & $\mathrm{n} / \mathrm{a}$ & Transcription factor & Yes & Yes & [57] \\
\hline & CD34 & $\begin{array}{l}\text { Cluster of differentiation } \\
\text { molecule } 34\end{array}$ & Surface antigen & No & Yes & {$[58]$} \\
\hline & FLT1 & $\begin{array}{l}\text { Vascular endothelial } \\
\text { growth factor receptor } 1\end{array}$ & Surface receptor & Yes & Yes & {$[59]$} \\
\hline & RUNX1 & $\begin{array}{l}\text { AML1, Runt-related } \\
\text { transcription factor } 1\end{array}$ & Transcription factor & Yes & Yes & {$[60]$} \\
\hline
\end{tabular}

is also often used to demonstrate the lack of pluripotency genes upon EB differentiation, thus demonstrating the completeness of differentiation. This is of particular concern with hiPSCs, in order to demonstrate that the factors used to reprogram in the case of integrating retroviruses are in fact silenced [62]. There are several pluripotent and germ layer-specific markers used for the purpose of ascertaining differentiation within EBs (Table 2).

5.2. Histological Analysis of Embryoid Bodies. Though gene expression analysis can be used to identify the presence of germ layer-specific markers and the concomitant lose of pluripotent markers, a more definitive assessment of tissue differentiation is based on the histological evaluation of sectioned EBs followed by assessment of tissue organization, cellular morphology, and localized protein expression [16, $28,29,36,63]$. Typically, EBs are first pelleted and embedded (i.e., low-melting point agarose) in order to concentrate the EBs for subsequent paraffin embedding and sectioning for mounting on microscope slides [63]. These slide-mounted sections are then stained with hematoxylin-eosin (H\&E) in order to enhance contrast between the tissues and cells (Figure 2). Using these techniques, various tissues can be commonly recognized such as neural rosettes (ectodermFigures 2(a), 2(d), 2(g), and 2(j)), connective tissue (mesoderm-Figures 2(b), 2(e), 2(h), and 2(k)), and putative endoderm (Figures 2(c), 2(f), 2(i), and 2(l)). Though this technique is commonly used for both EBs and teratomas to determine the presence of germ layer representatives once their histomorphologies are identified, the assessment can 


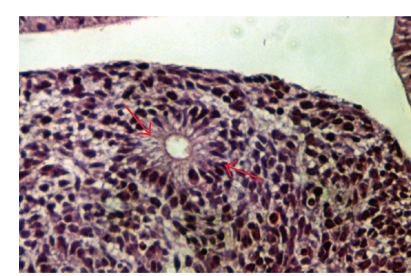

(a)

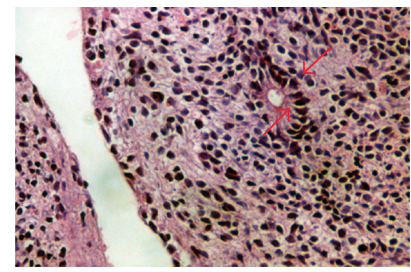

(d)

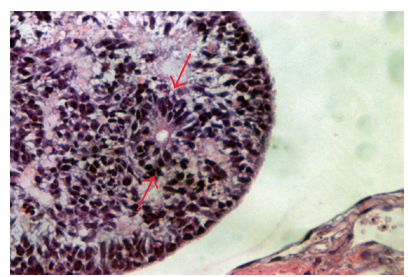

(g)

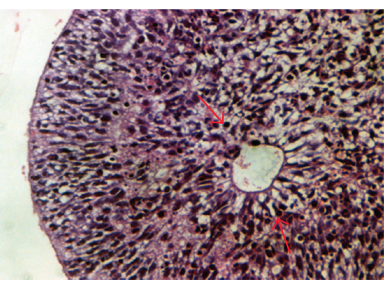

(j)

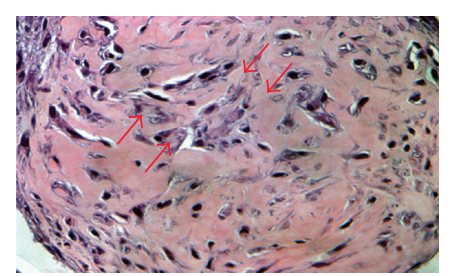

(b)

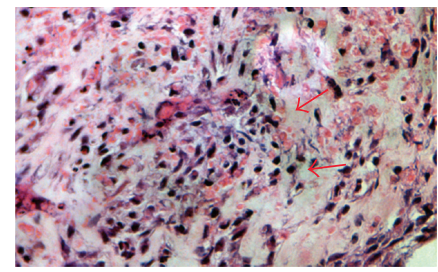

(e)

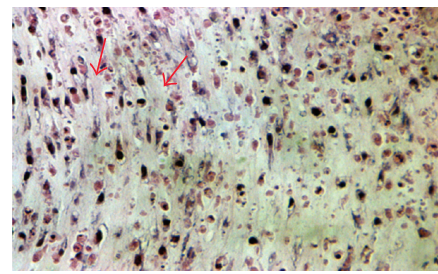

(h)

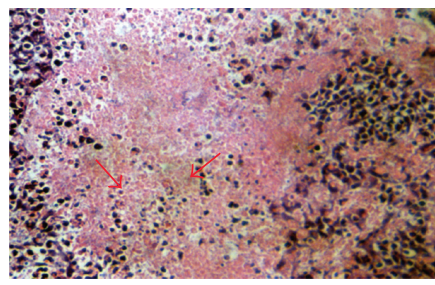

(k)

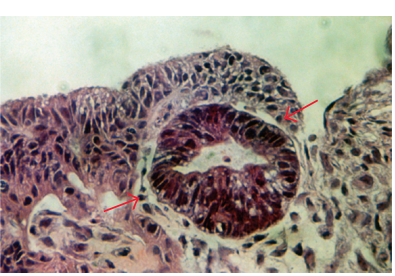

(c)

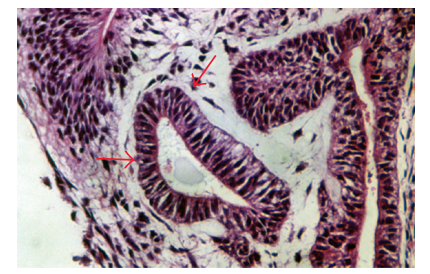

(f)

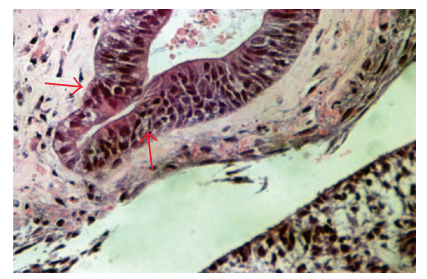

(i)

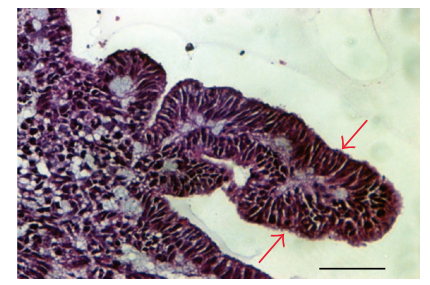

(1)

Figure 2: Histological evidence of germ layer differentiation in embryoid bodies generated from human pluripotent stem cells cultured by different methods to illustrate equivalence of culture techniques [63]. Shown are images of hematoxylin and eosin-stained histologic sections of EBs from hESCs propagated directly on mouse embryonic fibroblast (MEF) feeder layer (top row, a-c) or hESCs propagated in indirect coculture with MEFs (second row, $\mathrm{d}-\mathrm{f}$ ), hiPSCs propagated on MEFs (third row, g-i), or hiPSCs propagated in indirect coculture with MEFs (bottom row, $\mathrm{j}-1$ ). Equivalent trilineage potential is demonstrated by presence of ectodermal (neuroepithelial) (a, $d$, g, and $\mathrm{j}$ ); mesodermal (fibrous connective) (b, e, h, and k), and endodermal (intestinal) (c, f, i, and l) differentiation in these EBs. Arrows point to the corresponding tissue in each figure. Magnification is $400 \mathrm{x}$ total (10x ocular, 40x objective). Each scale bar represents $50 \mu \mathrm{m}$ in length.

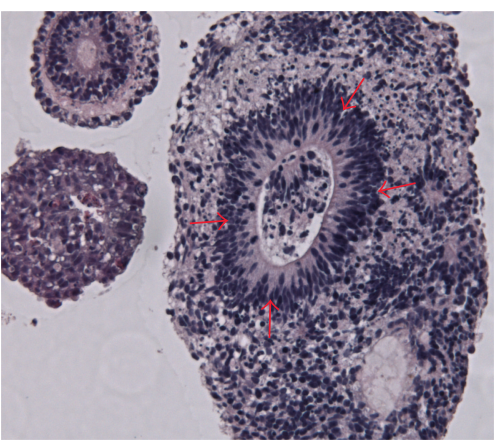

(a)

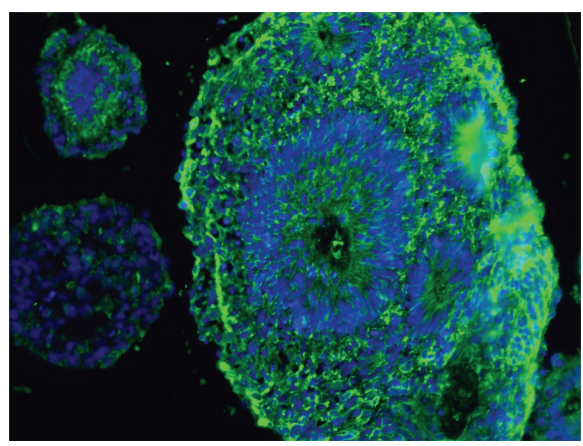

(b)

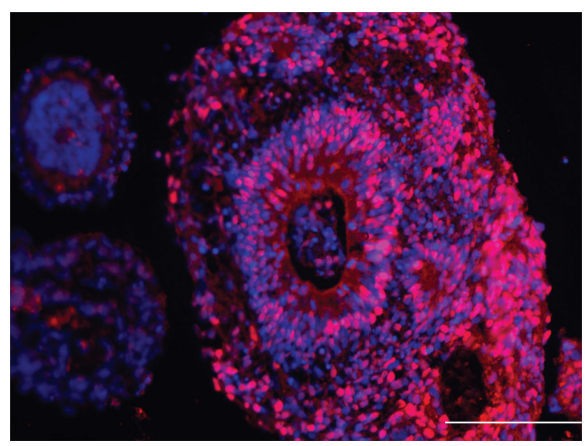

(c)

FIGURE 3: Immunohistochemical analysis of embryoid body sections confirming neuroepithelial tissue in adjacent sections, hematoxylin, and eosin-stained neural rosettes in hiPSCs-derived EBs (a), antinestin immunofluorescent staining (b), green-nestin, blue-nuclei stain, and anti-Sox2 immunofluorescent staining (c), red-Sox2, blue-nuclei stain. Magnification is 400x total (10x ocular, 40x objective). Each scale bar represents $50 \mu \mathrm{m}$ in length. 
be subjective and may require further immunohistochemical analysis in order to confirm the interpretation using specific antibodies (Table 2 and Figure 3 ) in sections adjacent to H\&E stained sections. This is particularly true of putative endoderm, which is less prevalent and typically less mature in embryoid bodies than the more straightforward identification of neural and connective tissues.

\section{Conclusions}

Although hiPSCs reprogrammed from human somatic cells have been well documented as a source of pluripotent stem cells with numerous shared similarities with hESCs, the increasing establishment of many new hiPSC lines requires the use of multiple assays and extensive resources to demonstrate their pluripotency on a line-by-line basis. Key among these is assays to demonstrate the potency towards formation of the three developmental germ layers and subsequent derivation of specific differentiated cell types in order to demonstrate their therapeutic potential. Recently, there has been enormous debate in the international stem cell community on the feasibility and use of the in vivo teratoma assay to demonstrate the pluripotency of derived hiPSCs $[4,6,8-$ 11]. Our paper proposes the embryoid body (EB) assay as a useful in vitro, cost-effective alternate to demonstrate the differentiation potential of derived hiPSCs. Methods for the generation of EBs and subsequent biochemical and histological analysis have gone through steady and tremendous improvements which permits its use across different laboratories. Further refinement and automation of the proposed methodologies provides opportunities for applications of the EB assay as a gold standard for assessing pluripotency of generated hiPSCs.

\section{Acknowledgment}

Partial funding for this work was provided by NSF-CAREER 074556 (R. Rao).

\section{References}

[1] S. M. Wu and K. Hochedlinger, "Harnessing the potential of induced pluripotent stem cells for regenerative medicine," Nature Cell Biology, vol. 13, no. 5, pp. 497-505, 2011.

[2] K. Takahashi, K. Tanabe, M. Ohnuki et al., "Induction of pluripotent stem cells from adult human fibroblasts by defined factors," Cell, vol. 131, no. 5, pp. 861-872, 2007.

[3] J. Yu, M. A. Vodyanik, K. Smuga-Otto et al., "Induced pluripotent stem cell lines derived from human somatic cells," Science, vol. 318, no. 5858, pp. 1917-1920, 2007.

[4] N. Maherali and K. Hochedlinger, "Guidelines and techniques for the generation of induced pluripotent stem cells," Cell Stem Cell, vol. 3, no. 6, pp. 595-605, 2008.

[5] K. Hochedlinger, "From MYOD1 to iPS cells," Nature Reviews Molecular Cell Biology, vol. 11, no. 12, p. 817, 2010.

[6] E. Dolgin, "Putting stem cells to the test," Nature Medicine, vol. 16, no. 12, pp. 1354-1357, 2010.

[7] R. Jaenisch and R. Young, "Stem cells, the molecular circuitry of pluripotency and nuclear reprogramming," Cell, vol. 132, no. 4, pp. 567-582, 2008.
[8] F. J. Müller, J. Goldmann, P. Löser, and J. F. Loring, "A call to standardize teratoma assays used to define human pluripotent cell lines," Cell Stem Cell, vol. 6, no. 5, pp. 412-414, 2010.

[9] C. Y. Fong, K. Gauthaman, and A. Bongso, "Teratomas from pluripotent stem cells: a clinical hurdle," Journal of Cellular Biochemistry, vol. 111, no. 4, pp. 769-781, 2010.

[10] G. Q. Daley, M. W. Lensch, R. Jaenisch, A. Meissner, K. Plath, and S. Yamanaka, "Broader implications of defining standards for the pluripotency of iPSCs," Cell Stem Cell, vol. 4, no. 3, pp. 200-201, 2009.

[11] J. Ellis, B. G. Bruneau, G. Keller et al., "Alternative induced pluripotent stem cell characterization criteria for in vitro applications," Cell Stem Cell, vol. 4, no. 3, pp. 198-199, 2009.

[12] I. H. Park, N. Arora, H. Huo et al., "Disease-specific induced pluripotent stem cells," Cell, vol. 134, no. 5, pp. 877-886, 2008.

[13] H. Kurosawa, "Methods for inducing embryoid body formation: in vitro differentiation system of embryonic stem cells," Journal of Bioscience and Bioengineering, vol. 103, no. 5, pp. 389-398, 2007.

[14] M. Koike, H. Kurosawa, and Y. Amano, "A round-bottom 96-well polystyrene plate coated with 2-methacryloyloxyethyl phosphorylcholine as an effective tool for embryoid body formation," Cytotechnology, vol. 47, no. 1-3, pp. 3-10, 2005.

[15] S. M. Dang, M. Kyba, R. Perlingeiro, G. Q. Daley, and P. W. Zandstra, "Efficiency of embryoid body formation and hematopoietic development from embryonic stem cells in different culture systems," Biotechnology and Bioengineering, vol. 78, no. 4, pp. 442-453, 2002.

[16] J. Itskovitz-Eldor, M. Schuldiner, D. Karsenti et al., "Differentiation of human embryonic stem cells into embryoid bodies compromising the three embryonic germ layers," Molecular Medicine, vol. 6, no. 2, pp. 88-95, 2000.

[17] S. Rungarunlert, M. Techakumphu, M. K. Pirity, and A. Dinnyes, "Embryoid body formation from embryonic and induced pluripotent stem cells: benefits of bioreactors," World Journal of Stem Cells, vol. 1, pp. 11-21, 2009.

[18] G. M. Keller, "In vitro differentiation of embryonic stem cells," Current Opinion in Cell Biology, vol. 7, no. 6, pp. 862-869, 1995.

[19] E. S. Ng, R. P. Davis, L. Azzola, E. G. Stanley, and A. G. Elefanty, "Forced aggregation of defined numbers of human embryonic stem cells into embryoid bodies fosters robust, reproducible hematopoietic differentiation," Blood, vol. 106, no. 5, pp. 1601-1603, 2005.

[20] A. H. Sathananthan, "Neural stem cells in neurospheres, embryoid bodies, and central nervous system of human embryos," Microscopy and Microanalysis, vol. 17, no. 4, pp. 520$527,2011$.

[21] M. Kumar, B. Bagchi, S. K. Gupta, A. S. Meena, P. Gressens, and S. Mani, "Neurospheres derived from human embryoid bodies treated with retinoic acid show an increase in nestin and Ngn2 expression that correlates with the proportion of tyrosine hydroxylase-positive cells," Stem Cells and Development, vol. 16, no. 4, pp. 667-681, 2007.

[22] J. C. Mohr, J. Zhang, S. M. Azarin et al., "The microwell control of embryoid body size in order to regulate cardiac differentiation of human embryonic stem cells," Biomaterials, vol. 31, no. 7, pp. 1885-1893, 2010.

[23] C. Y. Sargent, G. Y. Berguig, and T. C. McDevitt, "Cardiomyogenic differentiation of embryoid bodies is promoted by rotary orbital suspension culture," Tissue Engineering-Part A, vol. 15, no. 2, pp. 331-342, 2009.

[24] S. Takei, H. Ichikawa, K. Johkura et al., "Bone morphogenetic protein-4 promotes induction of cardiomyocytes from human 
embryonic stem cells in serum-based embryoid body development," American Journal of Physiology, vol. 296, no. 6, pp. H1793-H1803, 2009.

[25] P. W. Burridge, D. Anderson, H. Priddle et al., "Improved human embryonic stem cell embryoid body homogeneity and cardiomyocyte differentiation from a novel V-96 plate aggregation system highlights interline variability," Stem Cells, vol. 25, no. 4, pp. 929-938, 2007.

[26] B. Valamehr, S. J. Jonas, J. Polleux et al., "Hydrophobic surfaces for enhanced differentiation of embryonic stem cell-derived embryoid bodies," Proceedings of the National Academy of Sciences of the United States of America, vol. 105, no. 38, pp. 14459-14464, 2008.

[27] C. M. Cameron, W. S. Hu, and D. S. Kaufman, "Improved development of human embryonic stem cell-derived embryoid bodies by stirred vessel cultivation," Biotechnology and Bioengineering, vol. 94, no. 5, pp. 938-948, 2006.

[28] S. Gerecht-Nir, S. Cohen, and J. Itskovitz-Eldor, "Bioreactor cultivation enhances the efficiency of human embryoid body (hEB) formation and differentiation," Biotechnology and Bioengineering, vol. 86, no. 5, pp. 493-502, 2004.

[29] R. L. Carpenedo, C. Y. Sargent, and T. C. McDevitt, "Rotary suspension culture enhances the efficiency, yield, and homogeneity of embryoid body differentiation," Stem Cells, vol. 25, no. 9, pp. 2224-2234, 2007.

[30] B. S. Yoon, S. J. Yoo, J. E. Lee, S. You, H. T. Lee, and H. S. Yoon, "Enhanced differentiation of human embryonic stem cells into cardiomyocytes by combining hanging drop culture and 5-azacytidine treatment," Differentiation, vol. 74, no. 4, pp. 149-159, 2006.

[31] S. D. Sheridan, S. Gil, M. Wilgo, and A. Pitt, "Microporous membrane growth substrates for embryonic stem cell culture and differentiation," Methods in Cell Biology, vol. 86, pp. 2957, 2008.

[32] M. D. Ungrin, C. Joshi, A. Nica, C. Bauwens, and P. W. Zandstra, "Reproducible, ultra high-throughput formation of multicellular organization from single cell suspension-derived human embryonic stem cell aggregates," PLoS One, vol. 3, no. 2, Article ID e1565, 2008.

[33] C. Y. Fong, L. L. Chak, A. Subramanian et al., "A three dimensional anchorage independent In Vitro system for the prolonged growth of embryoid bodies to study cancer cell behaviour and anticancer agents," Stem Cell Reviews and Reports, vol. 5, no. 4, pp. 410-419, 2010.

[34] S. Gerecht, J. A. Burdick, L. S. Ferreira, S. A. Townsend, R. Langer, and G. Vunjak-Novakovic, "Hyaluronic acid hydrogel for controlled self-renewal and differentiation of human embryonic stem cells," Proceedings of the National Academy of Sciences of the United States of America, vol. 104, no. 27, pp. 11298-11303, 2007.

[35] K. Watanabe, M. Ueno, D. Kamiya et al., "A ROCK inhibitor permits survival of dissociated human embryonic stem cells," Nature Biotechnology, vol. 25, no. 6, pp. 681-686, 2007.

[36] M. L. M. Khoo, L. R. McQuade, M. S. R. Smith, J. G. Lees, K. S. Sidhu, and B. E. Tuch, "Growth and differentiation of embryoid bodies derived from human embryonic stem cells: effect of glucose and basic fibroblast growth factor," Biology of Reproduction, vol. 73, no. 6, pp. 1147-1156, 2005.

[37] B. R. Unsworth and P. I. Lelkes, "Growing tissues in microgravity," Nature Medicine, vol. 4, no. 8, pp. 901-907, 1998.

[38] P. I. Lelkes, D. L. Galvan, G. Thomas Hayman et al., "Simulated microgravity conditions enhance differentiation of cultured PC12 cells towards the neuroendocrine phenotype," In Vitro
Cellular and Developmental Biology-Animal, vol. 34, no. 4, pp. 316-325, 1998.

[39] S. Navran, "The application of low shear modeled microgravity to 3-D cell biology and tissue engineering," Biotechnology Annual Review, vol. 14, pp. 275-296, 2008.

[40] Y. Chisti, "Hydrodynamic damage to animal cells," Critical Reviews in Biotechnology, vol. 21, no. 2, pp. 67-110, 2001.

[41] T. G. Hammond and J. M. Hammond, "Optimized suspension culture: the rotating-wall vessel," American Journal of Physiology, vol. 281, no. 1, pp. F12-F25, 2001.

[42] J. Côme, X. Nissan, L. Aubry et al., "Improvement of culture conditions of human embryoid bodies using a controlled perfused and dialyzed bioreactor system," Tissue EngineeringPart C, vol. 14, no. 4, pp. 289-298, 2008.

[43] Y. S. Hwang, G. C. Bong, D. Ortmann, N. Hattori, H. C. Moeller, and A. Khademhosseinia, "Microwell-mediated control of embryoid body size regulates embryonic stem cell fate via differential expression of WNT5a and WNT11," Proceedings of the National Academy of Sciences of the United States of America, vol. 106, no. 40, pp. 16978-16983, 2009.

[44] M. K. Carpenter, E. Rosler, and M. S. Rao, "Characterization and differentiation of human embryonic stem cells," Cloning and Stem Cells, vol. 5, no. 1, pp. 79-88, 2003.

[45] F. Cavaleri and H. R. Schöler, "Nanog: a new recruit to the embryonic stem cell orchestra," Cell, vol. 113, no. 5, pp. 551$552,2003$.

[46] K. Adachi, H. Suemori, S. Yasuda, N. Nakatsuji, and E. Kawase, "Role of SOX2 in maintaining pluripotency of human embryonic stem cells," Genes to Cells, vol. 15, no. 5, pp. 455470, 2010.

[47] S. A. Przyborski, "Isolation of human embryonal carcinoma stem cells by immunomagnetic sorting," Stem Cells, vol. 19, no. 6, pp. 500-504, 2001.

[48] W. M. Schopperle and W. C. DeWolf, "The TRA-1-60 and TRA-1-81 human pluripotent stem cell markers are expressed on podocalyxin in embryonal carcinoma," Stem Cells, vol. 25, no. 3, pp. 723-730, 2007.

[49] E. Fuchs and K. Weber, "Intermediate filaments: structure, dynamics, function, and disease," Annual Review of Biochemistry, vol. 63, pp. 345-382, 1994.

[50] P. Callaerts, G. Halder, and W. J. Gehring, "Pax-6 in development and evolution," Annual Review of Neuroscience, vol. 20, pp. 483-532, 1997.

[51] E. Abranches, M. Silva, L. Pradier et al., "Neural differentiation of embryonic stem cells in vitro: a road map to neurogenesis in the embryo," PLoS One, vol. 4, no. 7, Article ID e6286, 2009.

[52] T. B. Tomasi Jr., "Structure and function of alpha-fetoprotein," Annual Review of Medicine, vol. 28, pp. 453-465, 1977.

[53] J. M. W. Slack, "Developmental biology of the pancreas," Development, vol. 121, no. 6, pp. 1569-1580, 1995.

[54] A. Mincheva, P. Lichter, G. Schütz, and K. H. Kaestner, "Assignment of the human genes for hepatocyte nuclear factor $3-\alpha,-\beta$, and $-\gamma$ (HNF3A, HNF3B, HNF3G) to 14q12-q13, 20p11, and 19q13.2-q13.4," Genomics, vol. 39, no. 3, pp. 417419, 1997.

[55] K. A. D’Amour, A. G. Bang, S. Eliazer et al., "Production of pancreatic hormone-expressing endocrine cells from human embryonic stem cells," Nature Biotechnology, vol. 24, no. 11, pp. 1392-1401, 2006.

[56] D. Durocher, F. Charron, R. Warren, R. J. Schwartz, and M. Nemer, "The cardiac transcription factors nkx2-5 and GATA4 are mutual cofactors," The EMBO Journal, vol. 16, no. 18, pp. 5687-5696, 1997. 
[57] M. Pekkanen-Mattila, M. Pelto-Huikko, V. Kujala et al., "Spatial and temporal expression pattern of germ layer markers during human embryonic stem cell differentiation in embryoid bodies," Histochemistry and Cell Biology, vol. 133, no. 5, pp. 595-606, 2010.

[58] A. B. Satterthwaite, T. C. Burn, M. M. Le Beau, and D. G. Tenen, "Structure of the gene encoding CD34, a human hematopoietic stem cell antigen," Genomics, vol. 12, no. 4, pp. 788794, 1992.

[59] T. V. Petrova, T. Makinen, and K. Alitalo, "Signaling via vascular endothelial growth factor receptors," Experimental Cell Research, vol. 253, no. 1, pp. 117-130, 1999.

[60] T. Okuda, J. Van Deursen, S. W. Hiebert, G. Grosveld, and J. R. Downing, "AML1, the target of multiple chromosomal translocations in human leukemia, is essential for normal fetal liver hematopoiesis," Cell, vol. 84, no. 2, pp. 321-330, 1996.

[61] B. Bhattacharya, J. Cai, Y. Luo et al., "Comparison of the gene expression profile of undifferentiated human embryonic stem cell lines and differentiating embryoid bodies," BMC Developmental Biology, vol. 5, article 22, 2005.

[62] T. Brambrink, R. Foreman, G. G. Welstead et al., "Sequential expression of pluripotency markers during direct reprogramming of mouse somatic cells," Cell Stem Cell, vol. 2, no. 2, pp. 151-159, 2008.

[63] S. Abraham, S. D. Sheridan, L. C. Laurent et al., "Propagation of human embryonic and induced pluripotent stem cells in an indirect co-culture system," Biochemical and Biophysical Research Communications, vol. 393, no. 2, pp. 211-216, 2010. 

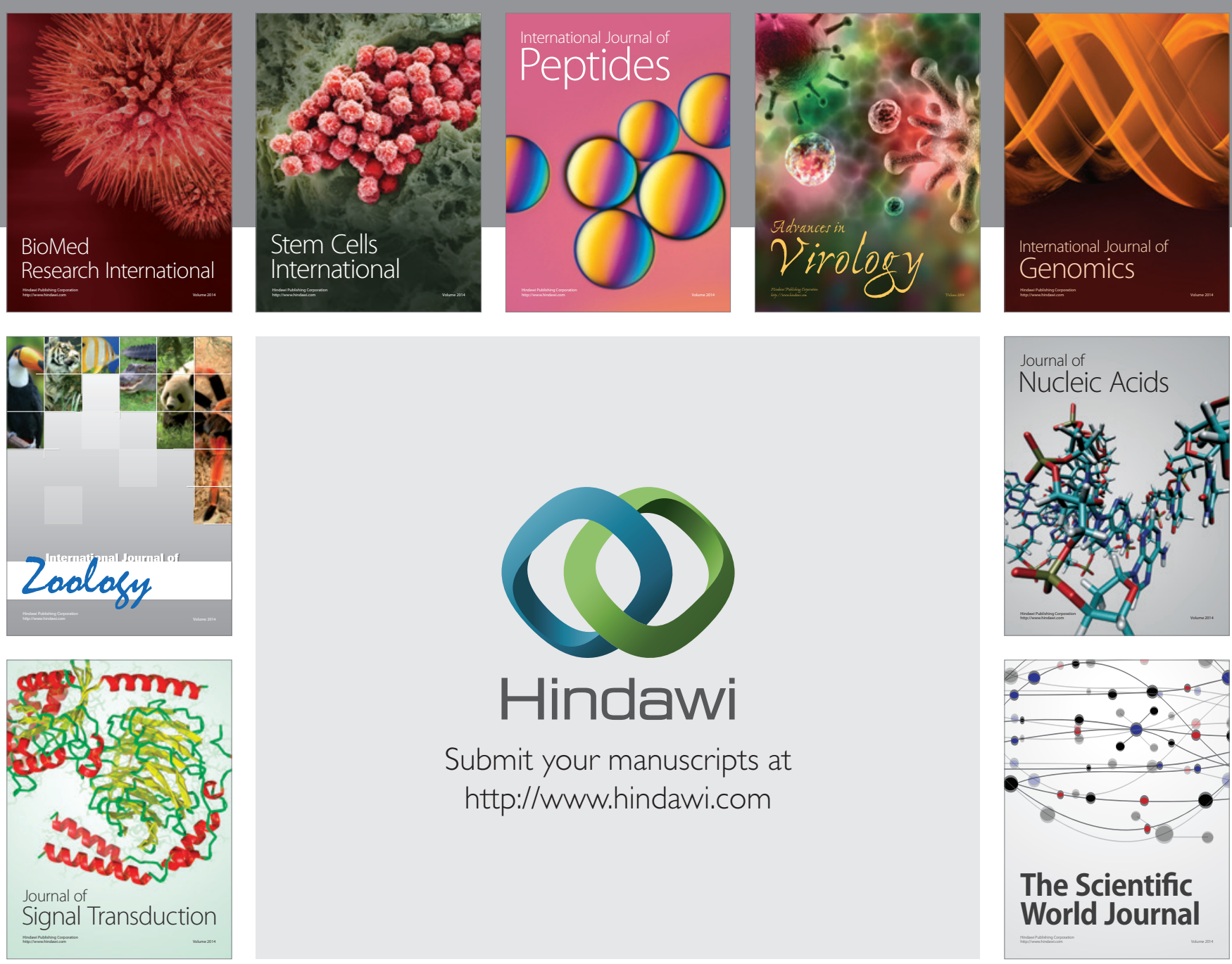

Submit your manuscripts at

http://www.hindawi.com
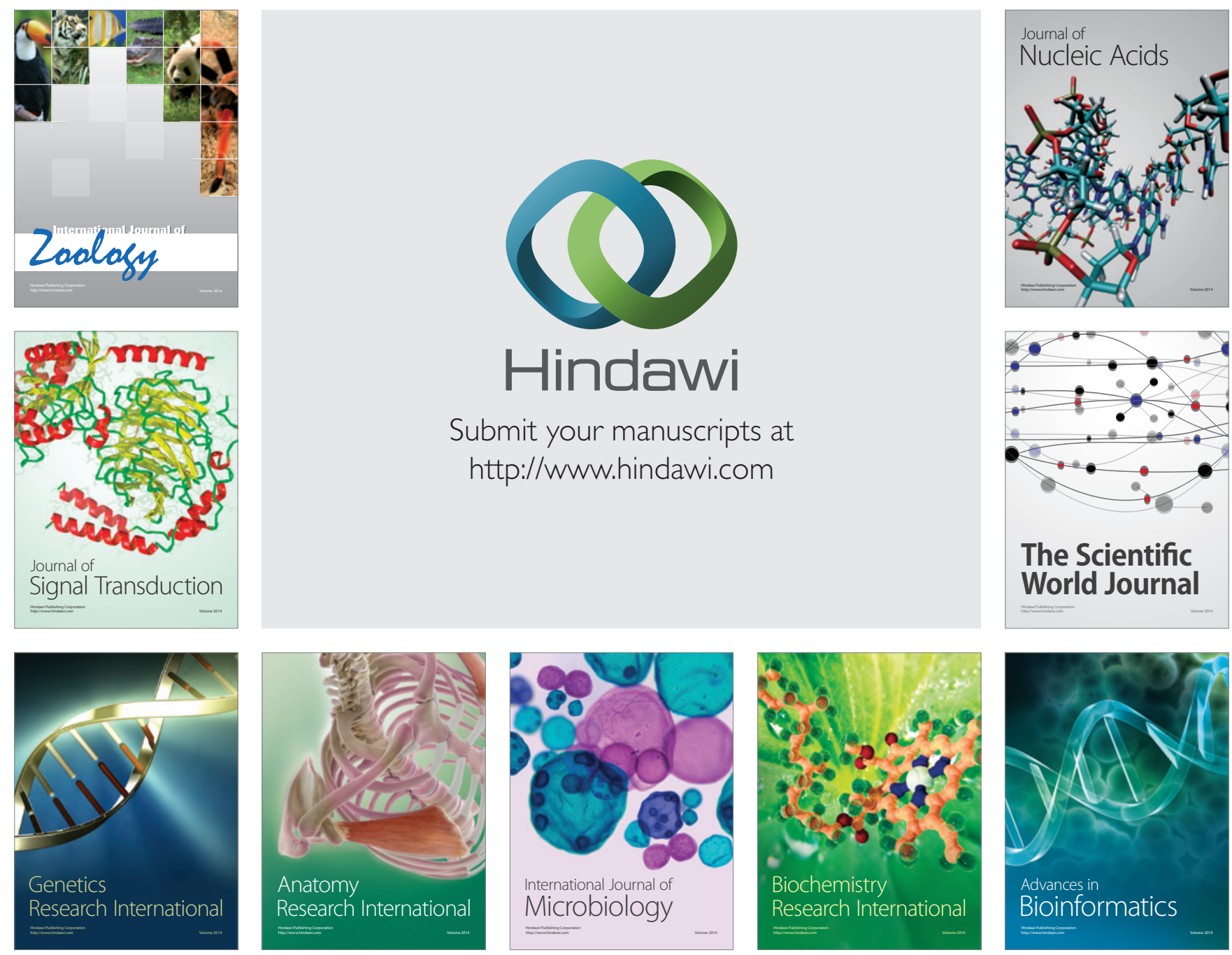

The Scientific World Journal
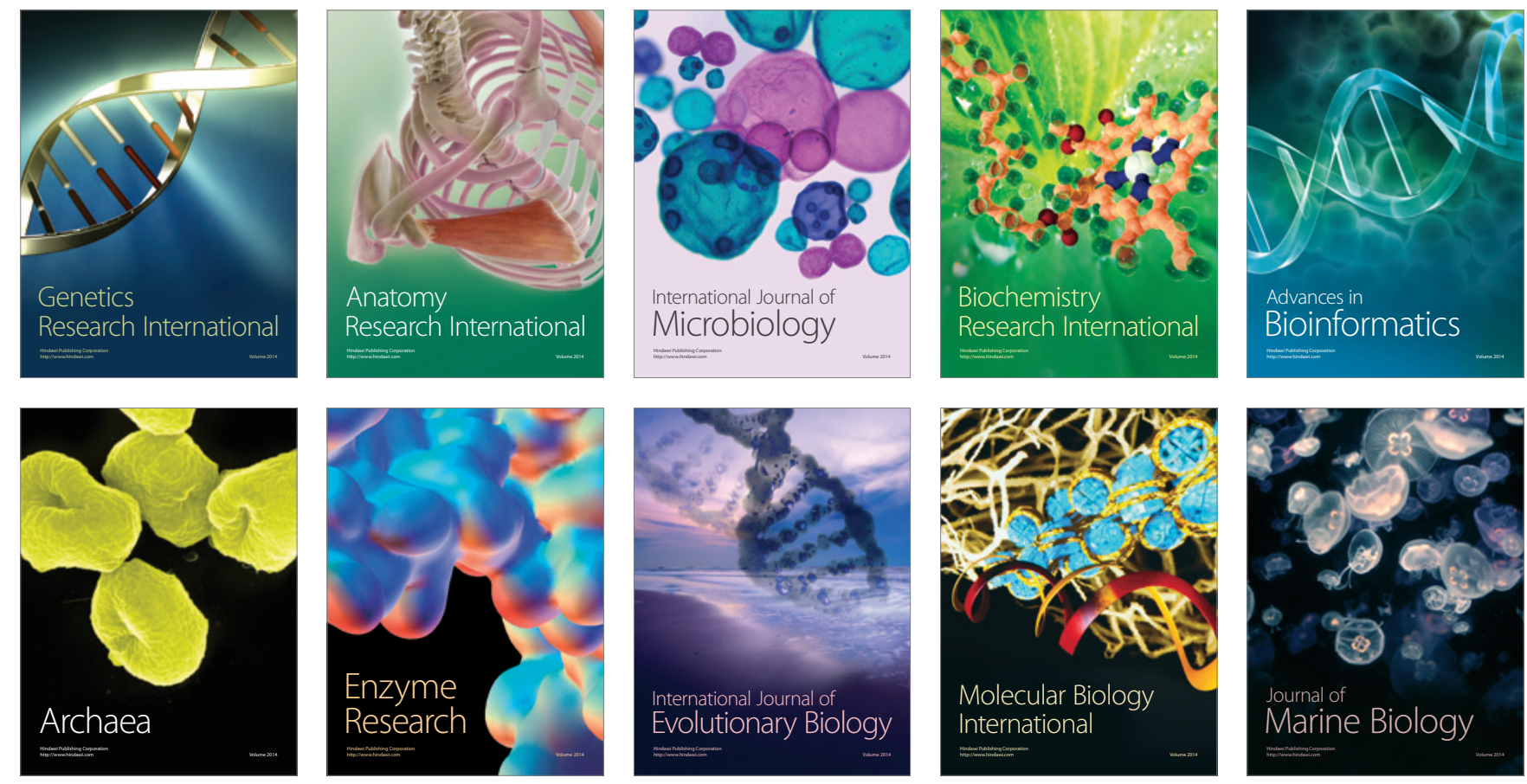